УДК

\title{
МОДЕЛЬ НА ПРОЦЕССЕ МАТЕРНА ДЛЯ ПОДАВЛЕНИЯ МЕЖСОТОВОЙ ИНТЕРФЕРЕНЦИИ В СИСТЕМАХ МНОГОСОТОВОЙ КООПЕРАЦИИ
}

\author{
С. ПРАТАП СИНГХ ${ }^{1}$, СУМАН ЯДАВ ${ }^{2}$, САНДЖЕЙ КУМАР \\ ${ }^{1}$ Технологический институт Бирль, \\ Индия, Джаркханд, 835215 \\ ${ }^{2}$ Галгошиаз инженерный и технологический колледж, \\ Индия, Нойда, Уттар-Прадеш
}

\begin{abstract}
Аннотация. Постоянно возрастающая потребность в скорости передачи данных и покрытии может быть достигнута преодолением недостатков беспроводных систем связи. Межсотовая интерференция ICI (inter cell interference) есть одним из наиболее сложно-преодолимых недостатков беспроводной связи. Для уменьшения влияния ICI в литературе предлагается техника, основанная на многосотовой кооперации MCC (multi cell cooperation). В этой статье уменьшение эффекта ICI достигается использованием процесса Матерна МНCP (Matérn Hard-Core Point Process) вместо пуассоновского процесса PРP (Poisson point process). В МНСР учитывается минимальное расстояние между базовыми станциями. Поэтому МНСР соответствует реальному сценарию размещения базовых станций. Также, использование МНСР существенно уменьшает аналитическую сложность в сравнении с РРР. Выражение в замкнутом виде для комплементарной интегральной функции распределения CCDF (complementary cumulative distribution function) отношения сигнал/помеха выведено с использованием МНCР для системы с ICI в зависимости от количества базовых станций, количества антенн и потерь распространения. Используя полученные выражения для $\mathrm{CCDF}$, аналитические выражения для функции плотности вероятности и эргодической пропускной способности также получены в замкнутом виде. В итоге анализ производительности системы выполнен в зависимости от эргодической пропускной способности. Полученные результаты согласуются с [4].
\end{abstract}

Ключевые слова: MHCP; CCDF; PDF; эргодическая пропускная способность; МСC

\section{1. ВВЕДЕНИЕ}

В литературе предлагаются различные техники преодоления недостатков беспроводной связи для обеспечения возрастающей потребности в скорости передачи данных. Среди других, межсотовая интерференция ICI (inter cell interference) есть одним из наиболее сложно-преодолимых недостатков систем беспроводной связи. Многосотовая кооперация МСС (multi cell cooperation) является одним из способов преодоления ICI $[1,2]$. Пуассоновский процесс PРP (Poisson point process) часто используется для моделирования расположения базовых станций (БС) в сотовых системах связи [3]. Однако, недавно предложено использовать процесс Матерна MHCP (Matérn Hard-Core Point Process) как альтернативу пуассоновскому [4]. Исследование использования МНСР вместо РРP для подавления ICI есть главной задачей этой статьи.

Стохастическая геометрия и схожие техники применяются в различных беспроводных сетях, таких как сотовые, когнитивные, радио-релейные с целью получения наиболее правдоподобного сценария $[5,6]$. МСС есть одной их техник, основанной на стохастической геометрии и кооперации между БС. Как 


\section{БИБЛИОГРАФИЧЕСКИЙ СПИСОК}

1. Huang, K.; Andrews, J. G. An analytical framework for multicell cooperation via stochastic geometry and large deviations. IEEE Trans. Inf. Theory, Vol. 59, No. 4, p. 2501-2516, 2012. DOI: 10.1109/TIT.2012.2232966.

2. Lee, N.; Morales-Jimenez, D.; Lozano, A.; Heath, R. W. Spectral efficiency of dynamic coordinated beamforming: A stochastic geometry approach. IEEE Trans. Wireless Commun., Vol. 14, No. 1, 2015. DOI: 10.1109/TWC.2014.2337305.

3. Sarkar, A.; Haenggi, M. Secrecy coverage. Internet Mathematics, Vol. 9, No. 2-3, p. 199-216, 2013. DOI: $10.1080 / 15427951.2012 .673333$.

4. Ibrahim, A. M.; Elbatt, T.; El-Keyi, A. Coverage probability analysis for wireless networks using repulsive point processes. Proc. of IEEE 24th Int. Symp. on Personal, Indoor and Mobile Radio Communications, 8-11 Sept. 2013, London UK. IEEE, 2013. DOI: 10.1109/PIMRC. 2013.6666284 .

5. Baccelli, F.; Giovanidis, A. A stochastic geometry framework for analyzing pairwise-cooperative cellular networks. IEEE Trans. Wireless Commun., Vol. 14, No. 2, p. 794-808, 2015. DOI: 10.1109/TWC.2014.2360196.

6. Haenggi, M.; Andrews, J. G.; Baccelli, F.; Dousse, O.; Franceschetti, M. Stochastic geometry and random graphs for the analysis and design of wireless networks. IEEE J. Selected Areas Commun., Vol. 27, No. 7, 2009. DOI: $10.1109 /$ JSAC.2009.090902.
7. Burchardt, H.; Haas, H. Multicell cooperation: evolution of coordination and cooperation in large-scale networks. IEEE Wireless Commun., Vol. 20, No. 1, p. 19-26, 2013. DOI: $10.1109 /$ MWC.2013.6472195.

8. Alfano, G.; Garetto, M.; Leonardi, E. New insights into the stochastic geometry analysis of dense CSMA networks. Proc. IEEE INFOCOM, 10-15 Apr. 2011, Shanghai, China. IEEE, 2011. DOI: 10.1109/INFCOM. 2011.5935092.

9. Stoyan, D.; Stoyan, H. On one of Matérn's hard-core point process models. Mathematische Nachrichten, Vol. 122, No. 1, p. 205-214, 1985. DOI: 10.1002/mana.19851220121.

10. Jones, M. O. Limiting behaviour of some spatial particle systems. Stochastic Models, Vol. 31, No. 2, p. 208-225, 2015. DOI: 10.1080/15326349.2014.994367.

11. Yadav, S.; Singh, S. Pratap. Novel closed form expression for multi cell cooperation using MHCP to reduce interference. Proc. of IEEE 18th Int. Symp. on Wireless Personal Multimedia Communications, 2015.

12. Yadav, S.; Singh, S. Pratap. Closed form expression of ergodic capacity for multi cell cooperation using MHCP. Proc. of IEEE UP Section Conf. on Electrical Computer and Electronics, UPCON, 4-6 Dec. 2015, Allahabad, India. IEEE, 2016. DOI: 10.1109/UP CON.2015.7456745.

13. Gradshteyn, I. S.; Ryzhik, I. M. Table of Integrals, Series, and Products, 7th ed. Academic Press Pub., 2007.

14. Hoydis, J.; Petrova, M.; Mahonen, P. Effects of topology on local throughput-capacity of ad hoc networks. Proc. of IEEE 19th Int. Symp. on Personal, Indoor and Mobile Radio Communications, 15-18 Sept. 2008, Cannes, France. IEEE, 2008. DOI: 10.1109/PIMRC.2008. 4699831. 